Portland State University

PDXScholar

Electrical and Computer Engineering Faculty

Publications and Presentations

Electrical and Computer Engineering

8-16-2007

\title{
Particle Shape as Revealed by Spectral \\ Depolarization
}

Donald D. Duncan

Portland State University

Michael Eugene Thomas

Johns Hopkins University

Follow this and additional works at: https://pdxscholar.library.pdx.edu/ece_fac

Part of the Electrical and Computer Engineering Commons

Let us know how access to this document benefits you.

Citation Details

D. D. Duncan and M. E. Thomas, "Particle shape as revealed by spectral polarization," Applied Optics, V. 46, pp. 6185-6191 (2007).

This Article is brought to you for free and open access. It has been accepted for inclusion in Electrical and Computer Engineering Faculty Publications and Presentations by an authorized administrator of PDXScholar. Please contact us if we can make this document more accessible: pdxscholar@pdx.edu. 


\title{
Particle shape as revealed by spectral depolarization
}

\author{
Donald D. Duncan ${ }^{1, \star}$ and Michael E. Thomas ${ }^{2}$ \\ ${ }^{1}$ Department of Biomedical Engineering, Oregon Health \& Science University, 3303 SW Bond Avenue, \\ Portland, Oregon 97239, USA \\ ${ }^{2}$ Applied Physics Laboratory, Johns Hopkins University, 11100 Johns Hopkins Road, Laurel, Maryland 20723, USA \\ ${ }^{*}$ Corresponding author: donald.duncan@bme.ogi.edu
}

Received 6 April 2007; revised 11 June 2007; accepted 15 June 2007; posted 19 June 2007 (Doc. ID 81895); published 16 August 2007

\begin{abstract}
Through a series of numerical simulations we explore some scatter effects due to nonspherical particles. Specifically, we examine the link between the aspect ratio of randomly oriented, prolate spheroidal particles and the resulting linear depolarization of the scattered light in the forward and backscatter directions. The particular objective is to detect the presence of randomly oriented particles that have a systematic size and aspect ratio. Calculations show that the spectral behavior of the linear depolarization reveals the aspect ratio of the scattering particles. The concept is demonstrated using the size, shape, and refractive index of the spore form of Bacillus globigii (BG). C 2007 Optical Society of America

OCIS codes: $290.1310,290.1350,290.5850,280.1100,280.1310,280.3640$.
\end{abstract}

\section{Introduction and Background}

A problem of contemporary interest is the detection of airborne biological agents that may constitute a health threat [1]. Our ultimate objective with this study is to suggest a remote means by which this might be accomplished. The concept relies upon what is commonly referred to as shape or structure birefringence [2]; the observed birefringence is due to the object's morphology rather than its molecular makeup.

It is well known that the angular distribution of scattered light reveals structural information about the scatterers. This is a practical measurement strategy when a diversity of scatter angles is accessible, as in flow cytometry $[3,4]$. For measurement configurations in which the viewing geometry is more restricted, however, other physical observables must be exploited for inference of such structural information. As an example, it has been known for some time that the polarization state of scattered light can reveal information about the structure of the individual scatterers. For instance, polarimetric lidars used in the remote sensing community differentiate between water and ice particles based on the spherical droplet's preservation of the polarization [5-7]; a nonzero

0003-6935/07/246185-07\$15.00/0

(C) 2007 Optical Society of America cross-polarized return is indicative of nonspherical scatterers. As a further example, polarimetric measurements of scatter from cometary dust [8] reveals information on the symmetry of the scatterers. For unpolarized solar illumination, circularly polarized scatter has been ascribed to the presence of asymmetric scatterers [9]. In the biomedical realm, Bickel et al. [10] used polarization measurements to differentiate between two strains of Bacillus subtilis spores having structural differences. Their measurements were at a single wavelength and provided a means of differentiation between the two bacteria strains but did not reveal the relationship between the morphological differences and the polarization state. Other workers [11,12] noted spectral differences in the polarization of light scattered by spheroidal particles but similarly did not draw a direct link between shape and wavelength dependence of the polarization. More recently, Mischenko and Hovenier [13] explored the relationship between depolarization and the particle's aspect ratio and size parameter. They dealt with the forward problem in which the depolarization was shown to vary in a periodic fashion with size parameter but did not discuss the inverse problem in which this variation might be exploited to infer the aspect ratio. In contrast, we demonstrate through a series of numerical simulations that it is possible to derive detailed shape information from the state of depolarization of the 
scattered light. The simulations are appropriate for particle suspensions in liquid or air.

In describing the effects of a suspension of scatterers, we make use of the Stokes-Mueller formulation. In this formalism, light is described in terms of a Stokes vector:

$$
\mathbf{I}=\left|\begin{array}{c}
I \\
Q \\
U \\
V
\end{array}\right|
$$

where the individual components of the vector are given by

$$
\begin{aligned}
& I=\left|E_{\|}\right|^{2}+\left|E_{\perp}\right|^{2}, \\
& Q=\left|E_{\|}\right|^{2}-\left|E_{\perp}\right|^{2}, \\
& U=E_{\|} E_{\perp} *+E_{\perp} E_{\|}^{*}, \\
& V=i\left(E_{\|} E_{\perp} *-E_{\perp} E_{\|}^{*}\right),
\end{aligned}
$$

and $E_{\|}$and $E_{\perp}$, respectively, are the electric field components parallel and perpendicular to the scatter plane. For a monodisperse aerosol, scatter is described by the equation [14]

$$
\mathbf{I}_{s c a}=\frac{C_{s c a} N d v}{4 \pi R^{2}} \mathbf{F}(\theta) \mathbf{I}_{i n c}
$$

where $C_{s c a}$ is the scatter cross section, $N$ is the particle number density, $d v$ is the volume element, $R$ is the distance from the volume element, $I_{i n c}$ and $I_{s c a}$, respectively, are the Stokes vectors for the incident and scattered radiation, and $\mathbf{F}(\theta)$ is the (angledependent) Mueller matrix. For an assembly of randomly oriented particles, each of which has a plane of symmetry (e.g., finite cylinders) and if the particles and their mirror images are in equal numbers and with random orientation then the Mueller matrix is given by [15]

$$
\mathbf{F}(\theta)=\left|\begin{array}{cccc}
F_{11}(\theta) & F_{12}(\theta) & 0 & 0 \\
F_{12}(\theta) & F_{22}(\theta) & 0 & 0 \\
0 & 0 & F_{33}(\theta) & F_{34}(\theta) \\
0 & 0 & -F_{34}(\theta) & F_{44}(\theta)
\end{array}\right|
$$

To quantify the phenomenon of interest, we adopt a common (although not unique, e.g., [13]) definition of the linear depolarization [12]:

$$
D(\theta)=1-F_{22}(\theta) / F_{11}(\theta) .
$$

Note that for spheres, the corresponding Mueller matrix is

$$
\mathbf{F}(\theta)=\left|\begin{array}{cccc}
F_{11}(\theta) & F_{12}(\theta) & 0 & 0 \\
F_{12}(\theta) & F_{11}(\theta) & 0 & 0 \\
0 & 0 & F_{33}(\theta) & F_{34}(\theta) \\
0 & 0 & -F_{34}(\theta) & F_{33}(\theta)
\end{array}\right|,
$$

and as a result, the linear depolarization is identically zero. Due to the symmetry of such a particle, there is no depolarization. In Section 2, we characterize wavelength dependence of this linear depolarization in the forward, $D(0)$, and backscatter, $D(\pi)$, directions.

\section{Calculations and Results}

For the study, we made use of publicly available FORTRAN codes for $T$-matrix calculations $[16,17]$. We will not dwell on the details of this algorithm as many good discussions exist in the literature (see, for example, Nilsson et al. [18]), and the validity of this particular code is well accepted [19]. Calculations were performed for a monodisperse aerosol of particle radius, $r=0.425 \mu \mathrm{m}$ over the wavelength interval $(0.4,1.6) \mu \mathrm{m}$. We assumed randomly oriented prolate spheroidal particles of major and minor axes $a$ and $b$, respectively (Fig. 1), and the aspect ratio, $\varepsilon=a / b$, was varied over the interval $(1,3)$ while keeping the particle volume constant:

$$
V=\frac{4}{3} \pi r^{3}=\frac{4}{3} \pi a b^{2} .
$$

The major and minor axes in terms of the equivalent volume radius and the aspect ratio are thus

$$
\begin{aligned}
& a=\varepsilon^{2 / 3} r, \\
& b=\varepsilon^{-1 / 3} r .
\end{aligned}
$$

Spore form Bacillus globigii (BG) has an aspect ratio on the order of 2 , but we performed calculations over a broader aspect interval to illustrate the effect that this parameter has on the depolarization signature.

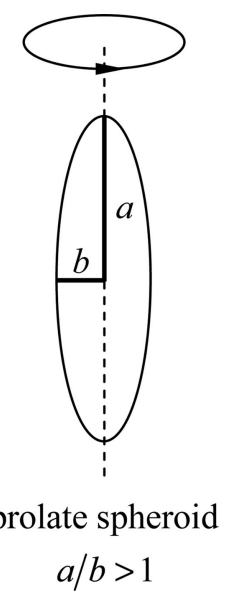

Fig. 1. Spheroidal particle illustration. 


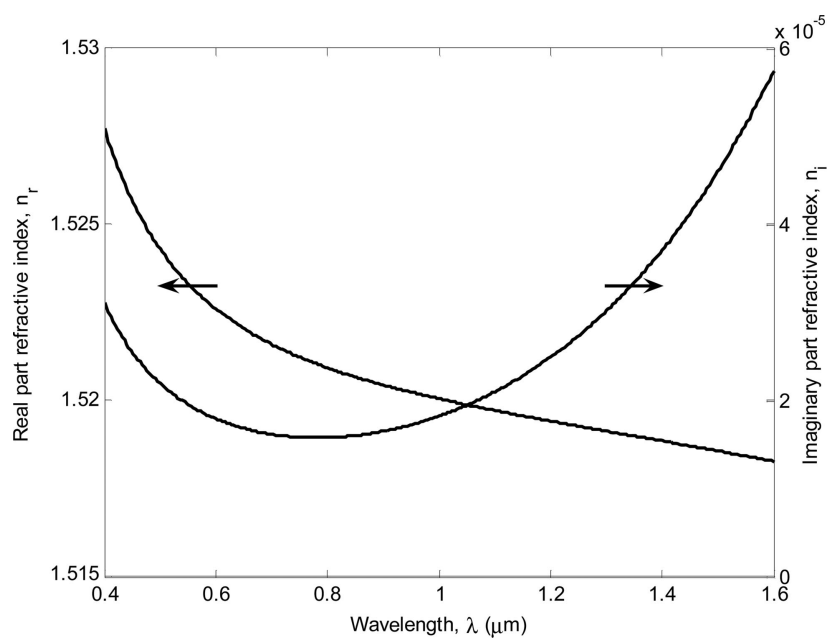

Fig. 2. Complex refractive index for BG.

To complete the specification of the optical properties of $\mathrm{BG}$, we require the spectral complex refractive index. Such a specification, based on data from the literature [20] along with spectrometer measurements of thin films of spores and a classical oscillator model [21], is shown in Fig. 2. In the following, we characterize the linear depolarization for monodispersions in the forward direction, the backscatter direction, and then explore the effects of differing particle radii.

\section{A. Forward-Scatter Direction}

With the previously discussed approach we arrived at estimates of the linear depolarization for the forwardscatter direction shown in Fig. 3. Our objective is to characterize the fine scale structure in these results in terms of the particle physical characteristics. Toward this end, we show in Fig. 4 a number of cuts through this surface. Results are parameterized on the dimensionless variable, $x=k r$, where $k$ is the free-space wavenumber $(k=2 \pi / \lambda)$ and $r$ is the equivalent volumetric particle radius [see Eqs. (6) and (7)]. Clearly, the fine scale structure in the depolarization

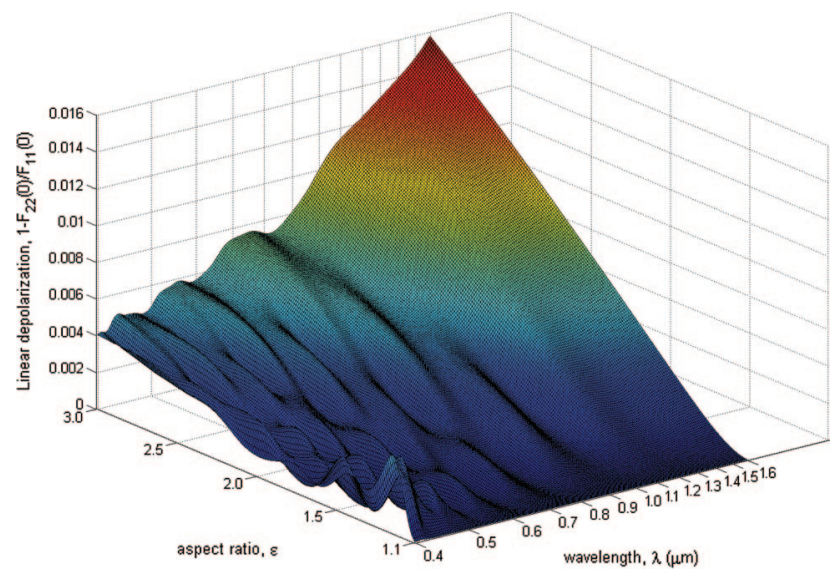

Fig. 3. (Color online) Linear depolarization in the forward direction as a function of wavelength and particle aspect ratio.

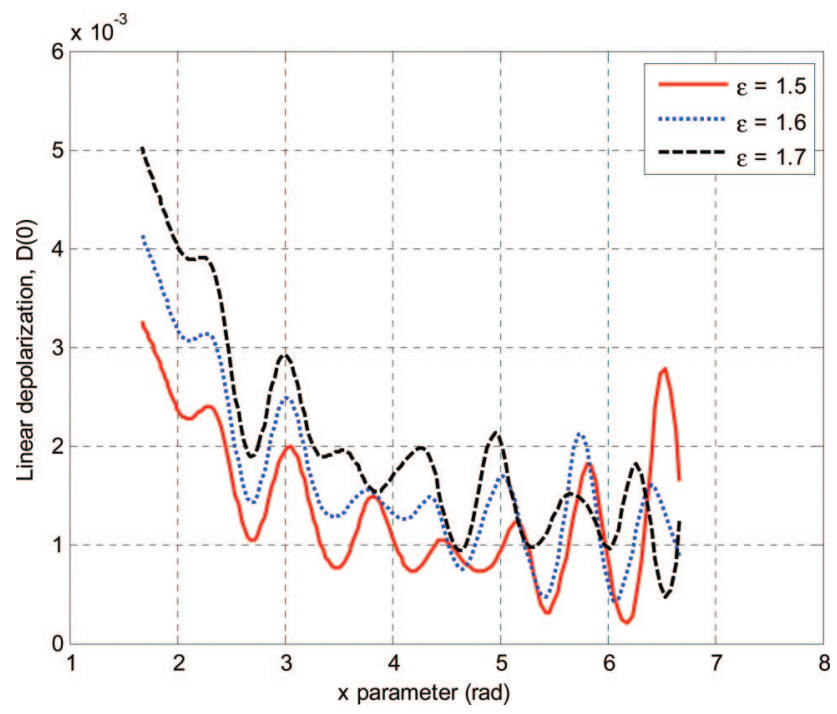

Fig. 4. (Color online) Illustration of fine scale structure in depolarization as a function of the $x$ parameter for various aspect ratios.

is evolving in response to the changing aspect ratio of the particles. We quantify this fine scale structure as illustrated in the following set of figures. Figure 5 is a plot of the depolarization for an aspect ratio of 1.5. Also shown in this figure is a least-squares fit of a fourth-order polynomial. We define the fine scale structure as the residual of this fit, as shown in Fig. 6 . Spectral analysis of this signal using a fast Fourier transform (FFT) yields the result shown in Fig. 7. In obtaining these results we used zero padding of the original array of 200 (wavelength) points to 4096 points and a Bartlett apodization window. Also indicated in this display are the two peak spectral components at 1.12 and $1.47 \mathrm{rad}^{-1}$. These peaks were determined automatically using the first numerical derivative and the sign of the second derivative. In this manner, true peaks were differentiated from in-

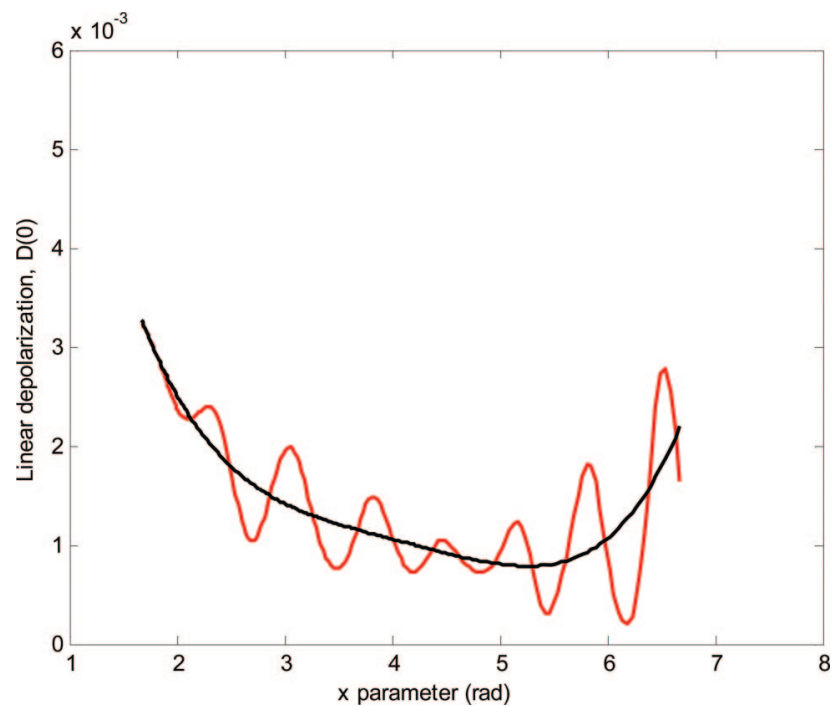

Fig. 5. (Color online) Linear depolarization for an aspect ratio of 1.5 and a fourth-order polynomial fit. 


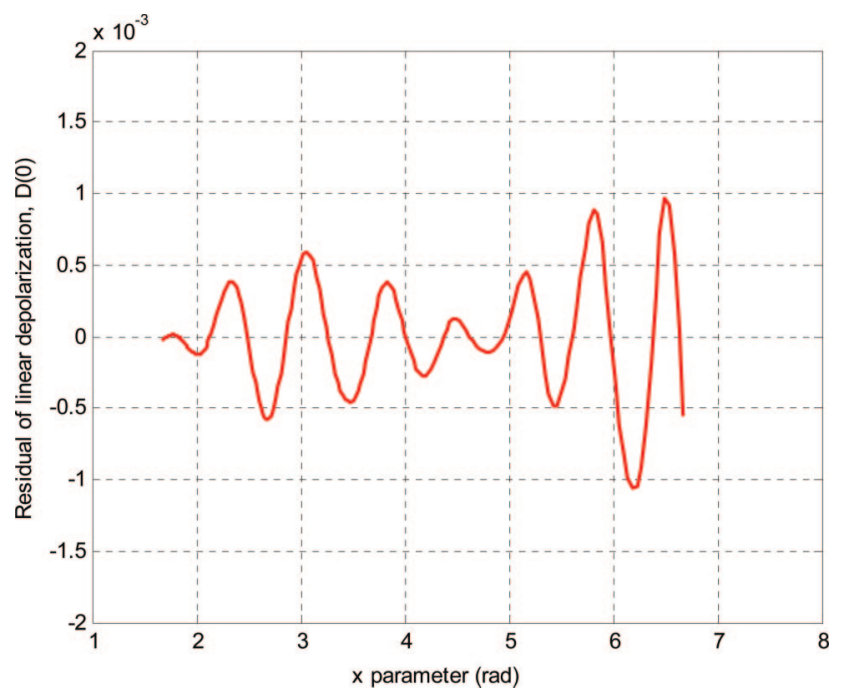

Fig. 6. (Color online) Residual of fit to depolarization for an aspect ratio of 1.5 .

flection points when the two spectral components were closely spaced. The periods of these two major components as a function of particle aspect ratio are shown in Fig. 8. Note that for an aspect ratio of unity the particles are spherical and the depolarization is identically zero. As one approaches unity aspect, the ratio $F_{22} / F_{11}$ approaches unity [see Eq. (4)], and as a result, limited numerical precision causes the estimate of depolarization to become noisy (because of the subtraction of two like numbers). That one period is increasing with increasing aspect ratio and the other is decreasing is highly suggestive in light of the fact that the major axis of the particle is increasing with increasing aspect ratio and the minor axis is decreasing. Clearly, the period of one spectral component is associated with the linear dimension of the major axis and one with the minor axis. Accordingly, we attempt a parameterization of this behavior in terms of the power-law fits:

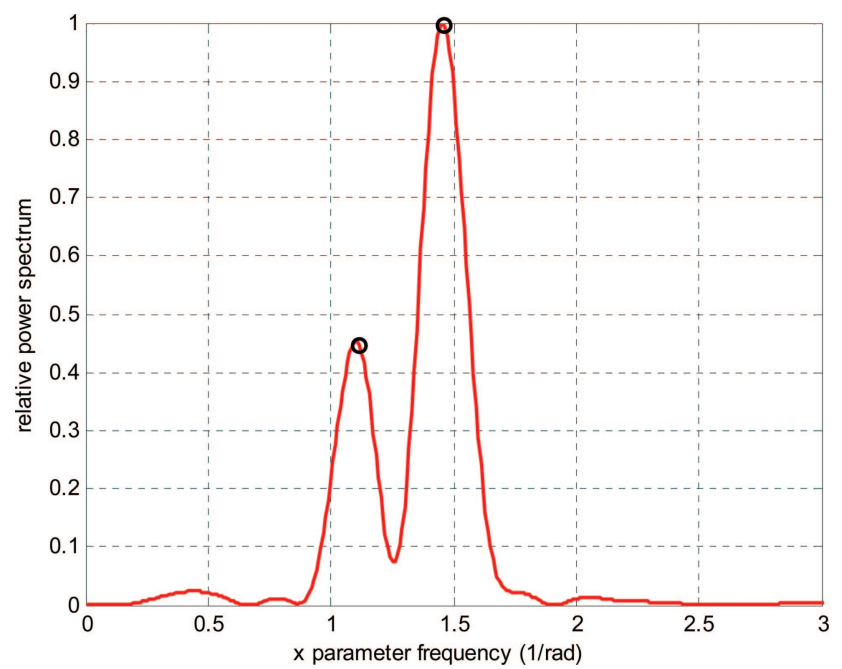

Fig. 7. (Color online) Frequency components of depolarization fine scale structure for an aspect ratio of 1.5.

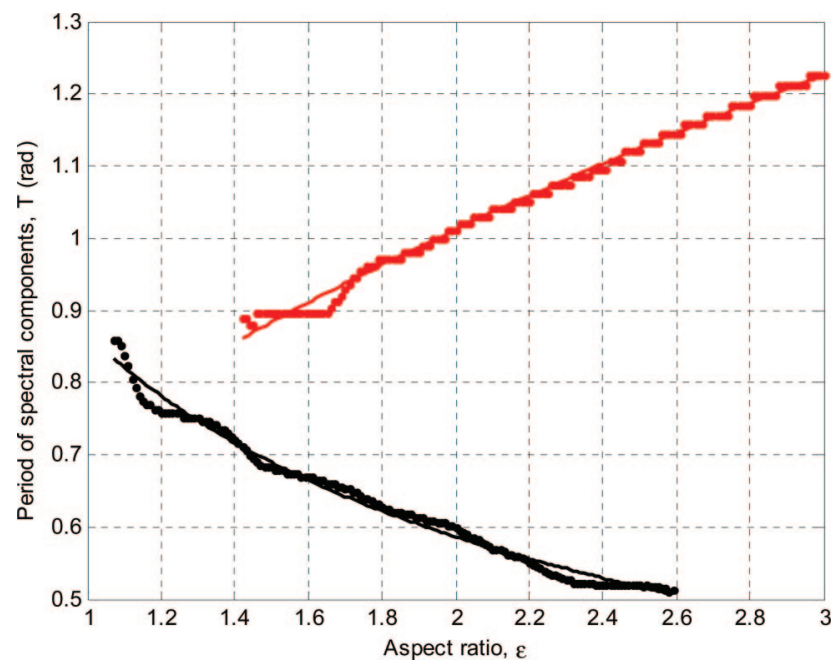

Fig. 8. (Color online) Periods of two major spectral components of depolarization fine scale structure as a function of particle aspect ratio and corresponding power-law fits.

$$
\begin{aligned}
& T_{a}=c_{a}(a / r)^{v_{a}}=c_{a}\left(\varepsilon^{2 / 3}\right)^{v_{a}}, \\
& T_{b}=c_{b}(b / r)^{v_{b}}=c_{b}\left(\varepsilon^{-1 / 3}\right)^{v_{b}} .
\end{aligned}
$$

Results of these fits are also illustrated in Fig. 8, and parameters of the fits are as follows:

$$
\begin{array}{ll}
c_{a}=0.30, & v_{a}=0.709, \\
c_{b}=0.866, & v_{b}=1.69 .
\end{array}
$$

These fits, in and of themselves, are not particularly interesting, but their ratio is revealing:

$$
T_{a} / T_{b}=\left(c_{a} / c_{b}\right) \varepsilon^{\left(2 v_{a} / 3\right)+\left(v_{b} / 3\right)}=0.842 \varepsilon^{1.04} .
$$

Alternatively, over the range of aspects for which two frequency components are observed, one could directly form the quotient of the periods and perform a least-squares fit on this quotient. These results suggest that a linear model is appropriate.

\section{B. Backscatter Direction}

Now if we inspect the linear depolarization in the backscatter direction we find the result shown in Fig. 9 . While the gross features of this result for the backscatter direction are quite distinct from forward scatter (Fig. 3), the fine scale structure displays similar behavior. This is borne out by the result shown in Fig. 10 , which also shows that the results of the powerlaw model fits to the data:

$$
\begin{array}{ll}
c_{a}=0.922, & v_{a}=0.709, \\
c_{b}=7.78, & v_{b}=4.23 .
\end{array}
$$

Based on these results, we find that the ratio of the two periods is given by

$$
T_{a} / T_{b}=\left(c_{a} / c_{b}\right) \varepsilon^{\left(2 v_{a} / 3\right)+\left(v_{b} / 3\right)}=0.118 \varepsilon^{1.88} .
$$




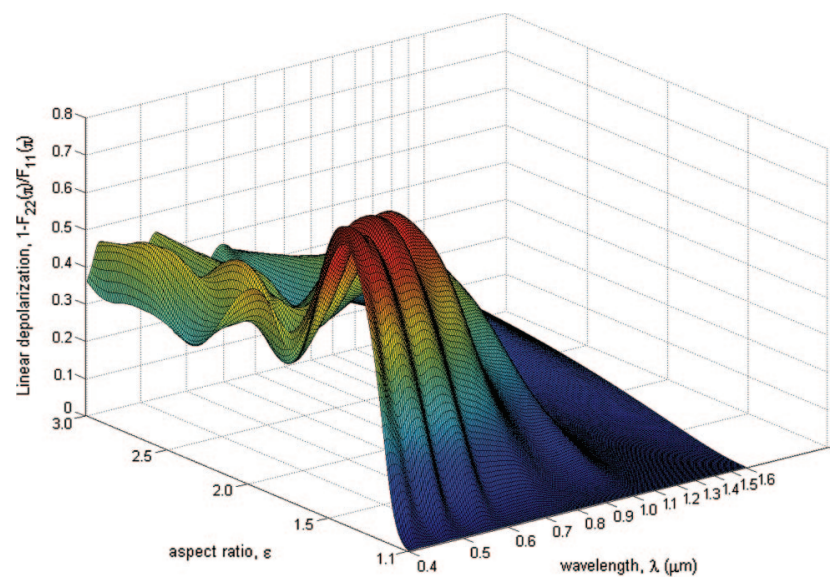

Fig. 9. (Color online) Linear depolarization in the backscatter direction as a function of wavelength and particle aspect ratio.

\section{Other Particle Radii}

Repeating the above procedure for other particle radii in the interval $(0.4,0.5) \mu \mathrm{m}$ yields the results in the forward-scatter direction:

$$
\begin{aligned}
T_{a} & =0.581 \varepsilon^{0.453} r^{-0.285}, \quad T_{b}=0.872 \varepsilon^{-0.516} r^{0.0335}, \\
T_{a} / T_{b} & =0.668 \varepsilon^{0.970} r^{-0.319},
\end{aligned}
$$

where the equivalent particle volumetric radius, $r$, is in micrometers. In the backscatter direction we find

$$
\begin{aligned}
T_{a} & =0.931 \varepsilon^{0.331} r^{-0.101}, \quad T_{b}=14.6 \varepsilon^{-1.66} r^{0.573}, \\
T_{a} / T_{b} & =0.0636 \varepsilon^{1.99} r^{-0.675} .
\end{aligned}
$$

As illustrated by Fig. 10, the periods associated with the minor axis of the particle tend to be on the order

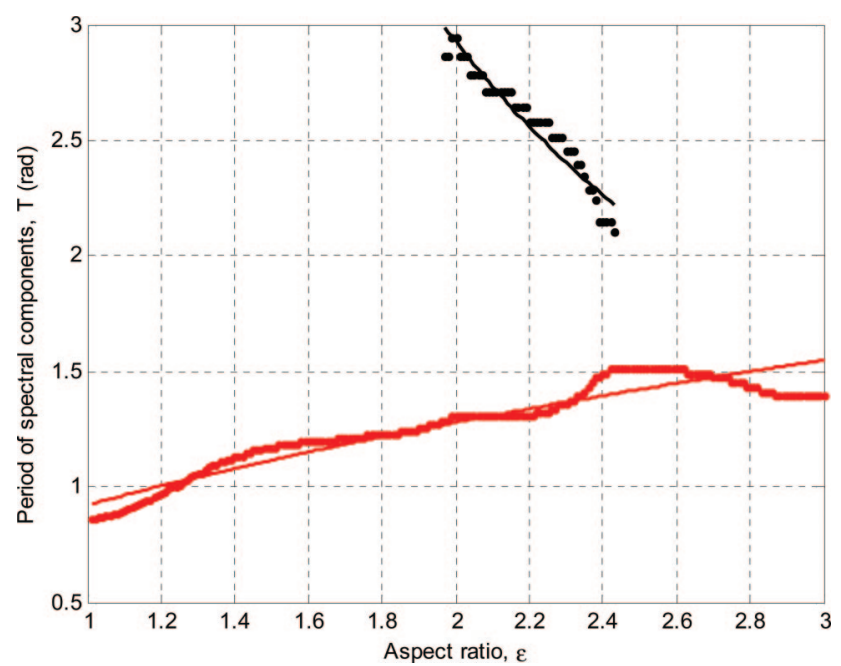

Fig. 10. (Color online) Periods of depolarization fine scale structure and power-law fits for backscatter. These results are analogous to those shown in Fig. 9. of 2-3 rad. Given that the entire $x$-parameter variation of the simulation is on the order of 5 rad (Fig. 6), the estimates of period associated with the backscatter regime are somewhat noisy. Moreover, this very low-frequency modulation is sensitive to details of the trend removal algorithm. This variability is manifested in the lack of fit, as seen in Fig. 10.

\section{Discussion and Conclusions}

Through a series of $T$-matrix calculations we have demonstrated that in principle, it is possible to determine the aspect ratio of randomly oriented, prolate spheroidal particles. This determination is based on inspection of the spectral behavior of the linear depolarization ratio. This is most easily accomplished in the forward-scatter regime where the quotient of the two major periods of the oscillatory behavior are linearly related to the aspect ratio of the scatterers and the dependence on the equivalent volumetric particle radius is weak. This dependence on particle radius is important as a real aerosol would display a range of sizes. The relationship shown in Eq. (13) allows one to calculate the effect of such a size distribution.

In the forward-scatter regime, linear dependence on aspect ratio makes sense on physical grounds. One might argue that for a particular ratio of $a: b$ (where $a$ and $b$, respectively, are the major and minor axes), there will be some frequency $(1 / \lambda)$ that will be optimal for coupling one polarization into the orthogonal polarization, i.e., a frequency that will result in an elevated depolarization. It makes sense that a harmonically related frequency will result in optimal depolarization for the corresponding aspect ratio $b: a$. In other words, as the frequency is swept, the depolarization will display a periodicity that is associated with a mode (i.e., polarization) conversion from $a \rightarrow b$, then $b \rightarrow a$, then $a \rightarrow b$, etc. It follows that the primary dependence of this periodicity must be on aspect ratio. As the aspect ratio increases, so too will the period of this ratio. This effect involves the distribution of the surface fields along the major and minor axes of the particle and is somewhat different from the oscillatory spectral variation in the scatter efficiency, which Bohren and Huffman [22] attribute to the interference between the undeviated ray and the ray through the center of the particle.

Interestingly, the quotient of the depolarization periods for the backscatter regime displays a quadratic dependence on aspect ratio. Further, the power-law dependence on particle size is nearly double that seen for forward scatter. That these power laws are doubled suggests that the depolarization in the backscatter regime is a higher-order effect.

Backscatter depolarization dependence on aspect ratio and $x$-parameter has been explored previously, e.g., [13], but for a fixed refractive index. For such a condition, variations in $x$ are effected by changing either the wavelength or the particle size. Note that these authors varied the particle aspect ratio while preserving surface area, not volume, as in the current study. 
As pointed out by Barnaba and Gobbi [23], this depolarization may be a useful discrimination tool. It can be used to differentiate between an aerosol with a consistent size and aspect ratio, and ambient aerosols. Ambient aerosols such as dust do depolarize the scattered light, but because these particles do not possess a well-defined shape, they will not generate a periodic depolarization signal [8]. Likewise, other ambient aerosols, such as pollens, which have a very complex shape and are substantially larger than visible wavelengths [24] are unlikely to produce such a depolarization modulation.

Mischenko [25] has noted that there are scatter angles for which the departure from unity of the ratio $F_{22} / F_{11}$ are larger, specifically a minimum of approximately $135-150^{\circ}$ and a maximum of approximately $160-165^{\circ}$. At these angles he found a strong dependence on particle shape. From an active remote sensing standpoint, however, the backscatter direction is more convenient. In such a case the transmitter and receiver can be colocated as in a typical lidar system. Further, the depolarization is stronger in the backscatter than the forward direction (compare Figs. 3 and 9). However, the depolarization is more slowly varying, and it may be more difficult to derive stable numerical estimates of its component periods. Although in principle it would be possible to perform a measurement over a wider wavelength interval and thus obtain more stable estimates of the periods, this is more difficult from an engineering standpoint. Another factor that is important in a practical remote sensing scheme is the susceptibility to measurement noise. Here again, the backscatter regime is to be preferred because of its much higher depolarization. These effects as well as more detailed signal processing algorithms deserve more study. As an example, based on the results shown here a parametric spectral analysis based on the a priori knowledge of only two spectral components may provide more stable estimates of the depolarization signature. Further work remains in optimizing the trend removal process prior to spectral analysis. Clearly there is a trade-off between the polynomial order and the lowest frequency one is attempting to resolve. Another variation on the study might be to explore the effect of aspect variation while preserving surface area instead of volume [13]. Of course, experimental verification of these predictions would be highly desirable.

As previously mentioned, this numerical study was intended to suggest a means of detecting the presence of a known pathogen, not as a formal estimation problem in which the morphology of an unknown biological species was to be determined. One could, however, envision the procedure's use in a classification scheme in which details of the spectral depolarization would allow for categorizing a number of different species. In any such detection system, however, it is important to be able to discriminate between scatter from a harmful species and that from background aerosols. On the basis of this study, we believe measurement of the spectral depolarization offers such a possibility.
The authors thank Scott Prahl of the Oregon Medical Laser Center for his insightful suggestions in the conduct of this research and his critical reading of the manuscript.

\section{References}

1. "Bioterrorism agents/diseases," Department of Health and Human Services, Centers for Disease Control and Prevention, http://www.bt.cdc.gov/Agent/agentlist.asp.

2. P. Vukusic, J. R. Sambles, and C. R. Lawrence, "Color mixing in wing scales of a butterfly," Nature 404, 457 (2000).

3. P. M. Pilarski and C. J. Backhouse, "A method for cytometric image parameterization," Opt. Express 14, 12720-12743 (2006).

4. J. D. Keener, K. J. Chalut, J. W. Pyhtila, and A. Wax, “Application of Mie theory to determine the structure of spheroidal scatterers in biological materials," Opt. Lett. 32, 1326-1328 (2007).

5. K. Sassen, "The polarization lidar technique for cloud research: a review and current assessment," Bull. Am. Meteorol. Soc. 72, 1848-1866 (1991).

6. A. Battaglia, O. Sturniolo, and F. Prodi, "Analysis of polarization radar returns from ice clouds," Atmos. Res. 49-60, 231250 (2001).

7. D. Müeller, U. Wandinger, D. Althausen, I. Mattis, and A. Ansmann, "Retrieval of physical particle properties from lidar observations of extinction and backscatter at multiple wavelengths," Appl. Opt. 37, 2260-2263 (1998).

8. L. Kolokolova, M. S. Hanner, A.-C. Levasseur-Regourd, and B. Å. S. Gustafson, "Physical properties of cometary dust from light scattering and thermal emission," in Comets II, M. C. Festou, H. U. Keller, and H. A. Weaver, eds. (University of Arizona Press, 2004).

9. D. Guirado, J. W. Hovenier, and F. Moreno, "Circular polarization of light scattered by asymmetrical particles," J. Quant. Spectrosc. Radiat. Transfer 106, 63-73 (2007).

10. W. S. Bickel, J. F. Davidson, D. R. Huffman, and R. Kolkson, "Application of polarization effects in light scattering: a new biophysical tool," Proc. Natl. Acad. Sci. U.S.A. 73, 486-490 (1976).

11. S. Asano, "Light scattering properties of spheroidal particles," Appl. Opt. 18, 712-723 (1979).

12. S. Asano and S. Makoto, "Light scattering by randomly oriented spheroidal particles," Appl. Opt. 19, 962-974 (1980).

13. M. I. Mischenko and J. W. Hovenier, "Depolarization of light backscattered by randomly oriented nonspherical particles," Opt. Let. 20, 1356-1359 (1995).

14. M. E. Thomas and D. D. Duncan, "Atmospheric transmission," in Atmospheric Propagation of Radiation, Volume 2 of the Infrared \& Electro-Optical Systems Handbook, F. G. Smith, ed. (ERIM Infrared Information Analysis Center and SPIE, 1993).

15. C. Brosseau, Fundamentals of Polarized Light: A Statistical Optics Approach (Wiley, 1998)

16. M. I. Mishchenko and L. D. Travis, "Capabilities and limitations of a current FORTRAN implementation of the $T$-matrix method for randomly oriented, rotationally symmetric scatterers," J. Quant. Spectrosc. Radiat. Transfer 60, 309-324 (1998).

17. FORTRAN $T$-matrix codes publicly available at http://www. giss.nasa.gov/ $\sim$ crmim.

18. A. M. K. Nilsson, P. Alsholm, A. Karlsson, and S. AnderssonEngels, " $T$-matrix computations of light scattering by red blood cells," Appl. Opt. 37, 2735-2748 (1998).

19. N. V. Voshchinnikov, V. B. Il'in, Th. Henning, B. Michel, and V. G. Farafonov, "Extinction and polarization of radiation by absorbing spheroids: shape/size effects and benchmark results," J. Quant. Spectrosc. Radiat. Transfer 65, 877-893 (2000). 
20. P. S. Tuminello, E. T. Arakawa, B. N. Khare, J. M. Wrobel, M. R. Querry, and M. E. Milham, "Optical properties of Bacillus subtilis spores from 0.2 to $2.5 \mu \mathrm{m}$,” Appl. Opt. 36, 2818-2824 (1997).

21. M. E. Thomas, M. B. Airola, N. T. Boggs, C. C. Carter, and J. E. Steinberg, "Complex refractive index of biological materials," in Proceedings of the 7th Joint Conference on Standoff Detection for Chemical and Biological Defense, Williamsburg, Virginia, USA (2006).

22. C. F. Bohren and D. R. Huffman, Absorption and Scattering of Light by Small Particles (Wiley-Interscience, 1983).
23. F. Barnaba and G. P. Gobbi, "Lidar estimation of tropospheric aerosol extinction, surface area and volume: Maritime and desert-dust cases," J. Geophys. Res. 106, 3005-3018 (2001).

24. L. D. Harder, "Pollen-size comparisons among animal pollinated angiosperms with different pollination characteristics," Biol. J. Linn. Soc. 64, 513-525 (1998).

25. M. I. Mischenko, "Light scattering by size-shape distributions of randomly oriented axially symmetric particles of a size comparable to a wavelength," Appl. Opt. 32, 4652-4666 (1993). 